lished: keeping oestrogen concentration to the lowest that is effective minimises that risk.

BLAIR H SMITH

Senior House Officer,

Department of Obstetrics and Gynaecology,

Northern General Hospital,

Sheffield S5 7AU

Professor of Obstetrics and Gynaecology,

Jessop Hospital for Women,

Sheffield S3 7RE

1 Schenker JG, Weinstein D. Ovarian hyperstimulation syndrome: a current survey. Fertil Ster 1978;30:255-68.

2 Karam KS, Taymor ML, Berger MJ. Estrogen monitoring and the prevention of ovarian overstimulation during gonadotrophin therapy. Am f Obstet Gynecol 1973;115:972-7.
3 McArdle C, Seibel M, Hann LE, Weinstein F, Taymor M. The diagnosis of ovarian hyperstimulation syndrome (OHS): the impact of ultrasound. Fertil Steril 1983;39:464-7.

Diamond MP, Wentz AC. Ovulation induction with human menopausal gonadotrophins. Obste Gynecol Surv 1986;41:480-90.

5 Navot D, Relou A, Birkenfield A, Rabinowitz R, Brzezinski A, Margalioth EJ. Risk factors and prognostic variables in the ovarian hyperstimulation syndrome. Am $\mathcal{F}$ Obstet Gynecol 1988;159: progn $210-5$

6 Paulson RJ, Do YS, Hsueh WA, Eggena P, Lobo TA. Ovarian renin production in vitro and in vivo: characterisation and clinical correlation. Fertil Steril 1989;51:634-8.

IAN D COOKE 7 Navot D, Margalioth EH, Laufer N, et al. Direct correlation between plasma renin activity and severity of the ovarian hyperstimulation syndrome. Fertil Steril 1987;48:57-61.

8 Tang GW, Chan SY. Further evaluation of first morning urinary oestrogens in monitoring gonadotrophin therapy. Aust N Z J Obstet Gynaecol 1988;28:141-3.

9 Balasch J, Carmona F, Llach J, Arroyo V, Jove I, Vanrell J. Acute prerenal failure and liver dysfunction in a patient with severe ovarian hyperstimulation syndrome (case report). Hum Reprod 1990;5:348-51.

10 Fishel S, Jackson P. Follicular stimulation for high tech pregnancies: Are we playing it safe? $B M$ 1989;299:309-11.

11 Cruikshank DJ. Aetiological importance of ovulation in epithelial ovarian cancer. BMf 1990;301 $524-5$

\title{
Leptospirosis update
}

\section{The risk is small and may be reduced by simple precautions}

Leptospirosis has recently been the subject of increased media attention, prompting confusion and anxiety among several groups who have not previously seen themselves as being at risk-such as canoeists and other recreational water users. What are the risks of contracting the disease, how dangerous is it, and can those at risk do anything to protect themselves?

The genus leptospira comprises two species: Leptospira biflexa, which includes saprophytic serovars (serotypes), and $L$ interrogans, which contains 202 pathogenic serovars in 23 serogroups. ${ }^{1}$ In the British Isles pathogenic leptospires from 10 serogroups have been isolated from wild and domestic animals. Infected animals usually remain well but excrete large numbers of organisms into the environment. Leptospires survive in moist conditions outside the host for many days (except in salt water, where they survive only a few hours) but are easily destroyed by drying, exposure to disinfectants and detergents, and heating to $50^{\circ} \mathrm{C}$ for five minutes. Humans become infected through contact with the urine or tissues of infected animals or through contact with water that harbours leptospires. Bacteria enter through skin cuts and abrasions or through the mucous membranes of the eyes or nasopharynx.

Leptospirosis presents in various ways, from inapparent to fulminating and fatal infection. After a seven to 12 day incubation period the disease has two overlapping phases, the first characterised by leptospiraemia and the second by leptospiruria and rising antibody titres. Turner's view in 1969 that "laboratory investigations will rarely help the patient because they can seldom confirm the diagnosis in time to influence treatment" remains true today. ${ }^{2}$ About $90 \%$ of symptomatic patients present with a mild influenza-like illness which usually resolves uneventfully in two to three weeks. Occasionally patients present with lymphocytic meningitis. Only a small proportion develop haemorrhagic complications and severe kidney and liver failure (Weil's disease), and only $10-20 \%$ of these patients die from myocarditis, haemorrhage, or multiorgan failure. Death without jaundice is virtually unknown, and chronic infection with persistent leptospiruria or uveitis is rare.

Leptospirosis is confirmed either by isolating the organism or, more commonly, by detecting specific antibodies in the patient's blood at the end of the first week of illness. Serological testing for leptospirosis is appropriate only for confirming an initial clinical suspicion and not for deciding whether or not to start treatment. Antibiotic treatment is controversial, and many people recover without treatment.

\section{Reducing the risk of leptospirosis}

- Eliminate rats and don't touch them with unprotected hands

- Cover all cuts with waterproof plasters and wear protective clothing

- Avoid immersion in natural waters

- Wear footwear

- Shower after canoeing, windsurfing, waterskiing, or swimming

Controlled trials suggest benefit by giving antibiotics within the first four days of illness-before the diagnosis can be confirmed. Severe cases need supportive care, and penicillin $1.5 \mathrm{MU}$ or ampicillin $1 \mathrm{~g}$ may be given parenterally every six hours. ${ }^{3}$ Alternatively, erythromycin $250 \mathrm{mg}$ every six hours or tetracycline $250 \mathrm{mg}$ every eight hours can be given to patients hypersensitive to penicillin.

Traditionally leptospirosis was associated with sewermen, miners, and fish workers, but farmers and agricultural workers are now the main occupational group at risk, accounting for $52 \%$ of cases. People in contact with rats or natural inland waters are potentially at risk-although this is small, and the data are inadequate to confirm whether recreational water users are at increased risk. Of the 299 confirmed cases in England and Wales during 1985-9, 268 occurred in the working age group 15-64 and 21 occurred in women. The disease has a well defined seasonal pattern with most cases occurring from July to December.

Leptospirosis due to $L$ sejroe serovar hardjo from cattle accounted for 157 of cases, L icterohaemorrhagiae for 87, undetermined serovars for 45 , and other serovars for 10 . The absence of human $L$ canicola infection since 1985 may be attributed to canine immunisation. Interestingly, six cases of $L$ sejroe serovar saxkoebing, which has recently been detected in cattle, were confirmed, with three cases related to water contact. During the five years there were 15 deaths, 11 due to $L$ icterohaemorrhagiae and four to undetermined strains (PHLS Communicable Disease Surveillance Centre, unpublished data). The Leptospira Reference Laboratory confirmed 44 cases from Scotland with one fatality, 42 cases from Ireland with four fatalities, and 92 cases acquired abroad during the same period.

Total prevention or eradication of leptospires (and hence absolute prevention of leptospirosis) is impossible. Large 
scale vaccination of domestic animals can prevent animal disease and renal excretion of leptospires. Human vaccines have been used overseas in high risk groups with varied success, but vaccines produced in rabbit serum may cause serious side effects. No human vaccine is available in the United Kingdom. The counsel of perfection is to avoid all contaminated waters, but simple precautions may reduce even further the small risk. Warning or safety cards containing the advice outlined in the box can be issued to people. There is no value in routine antibody screening for employment purposes. Should an individual become ill the safety card should be shown to his or her doctor, who can obtain advice from the microbiologist at the local hospital. In England and Wales public health laboratories offer diagnostic serological testing, and the Leptospira Reference Laboratory accepts serum samples for confirmation or from seriously ill patients in whom the diagnosis is in doubt.
Undoubtedly leptospirosis is underdiagnosed owing to its non-specific presentation, but probably few severe or fatal cases are missed. $L$ hardjo and $L$ icterohaemorrhagiae are the most common infecting serovars in the United Kingdom. Despite the recent reported increase in the rat population the increase in cases due to $L$ icterohaemorrhagiae in 1988 was not maintained in $1989 .{ }^{4}$

IAN R FERGUSON

Director,

Public Health Laboratory,

County Hospital,

Hereford HR1 2ER

1 International Union of Microbiological Societies Subcommittee on the Taxonomy of Leptospira. Revised list of Leptospira serovars. Gronogen: University Press, 1988.

Turner LH. Leptospirosis. BMF 1969;i:231-5.

3 Watt G, Padre LP, Tuazon L, et al. Placebo-controlled trial of intravenous penicillin for severe and late leptospirosis. Lancet 1988;i:433-3.

4 Golding C. Rats - the new plague. London: Weidenfield and Nicholson, 1990.

\section{Management of injuries due to chemical weapons}

\section{Most patients exposed to mustard gas recover completely}

The use (though not the possession) of chemical warfare agents is banned by international treaty. Nevertheless, Iraq has used these weapons recently and is likely to do so again if conflict breaks out in the Gulf. They were used most recently during the Iraq-Iran war, when Iraq used mustard gas (and possibly the nerve agent Tabun) against troops and Kurdish civilians. Some of these casualties were treated in London, so we have some recent experience of managing the victims of chemical weapons. What are the predominant agents? What injuries do they cause? and What is the outlook for their victims?

Since chemical weapons were first used on a large scale during the first world war a wide range has been developed, but only a small number of these are likely to be used by an aggressor. Because of their high volatility compounds such as hydrogen cyanide, phosgene, and chlorine are unlikely to be used. Mustard gas and nerve agents are likely to present the major risk.

Mustard gas (sulphur mustard) is a liquid which gives off a dangerous, visicant vapour. Unprotected individuals exposed to either the vapour or the liquid develop blistering of the skin, eye damage, and, if they inhale the vapour, damage to the upper respiratory tract. An asymptomatic latent period of up to six or so hours is classically described before features develop, an early reddening of the skin then progressing to blisters, which may be large and pendulous but tend not to be painful. Eye damage is commonly restricted to the anterior part of the eye, though severe pain, tearing, and corneal damage may take up to six weeks to resolve. Absorption of the compound may cause depression of the bone marrow, reaching a nadir some two weeks after exposure. Death, which in the first world war occurred in about $2 \%$ of mustard gas victims, results from burns, respiratory tract damage, and bone marrow depression.

Treatment of mustard gas exposure is according to symptoms. The skin lesions should be treated like thermal burns, large blisters being drained under aseptic conditions. The blister fluid in such cases is often claimed to be dangerous in itself, threatening secondary blistering of attendants: this is untrue. Damage to the eyes should be treated by daily irrigation; mydriatics to ease the eye pain produced by spasm of the ciliary muscle and to prevent the iris sticking to the lens; antibiotic drops; and, if necessary, systemic analgesics. The use of sterile petroleum jelly to prevent the lid margins sticking together is also recommended. Dark glasses and reassurance are very important as the eye lesions produce severe photophobia and fear. The value of local anaesthetics and steroid drops is more controversial, and they should not be used without expert advice.

Damage to the upper respiratory tract should be treated according to symptoms, and antibiotic cover provided to prevent infection. The most severely affected patients may need assisted ventilation and oxygen enriched air.

Most patients exposed to mustard gas recover completely, and only a small proportion will have long term eye or lung damage. Though sulphur mustard is a known human carcinogen, the risk associated with a single exposure is remote.

Nerve agents pose a more serious threat, particularly to the unprotected. They are organophosphorus compounds which inhibit the enzyme acetylcholinesterase and therefore interfere widely with the functioning of the nervous system. They are closely related to organophosphorus pesticides, and treatment of casualties exposed to these agents is broadly similar to the treatment of those poisoned with organophosphorus pesticides. Nerve agents may be encountered on the battlefield in both the vapour and liquid phases.

The early symptoms of nerve gas poisoning include miosis, rhinorrhoea, hypersalivation, and headache. In the most severely poisoned these may progress to impairment of neuromuscular transmission leading to respiratory failure. Vomiting, convulsions, and damage to the central respiratory drive may also occur, causing death from respiratory failure. Treatment includes the use of atropine, oximes (which reactivate the inhibited cholinesterase), and the anticonvulsant diazepam. The efficacy of treatment may be greatly enhanced by giving pyridostigmine before exposure to a nerve agent. Pyridostigmine binds reversibly to some of the cholinesterase and prevents that proportion being attacked by nerve agent. During the recovery period after poisoning with nerve agents binding of cholinesterase to pyridostigmine is reversed and uninhibited enzyme reappears from this protected store. Members of the United Kingdom armed forces have pyridos- 